\title{
A Distributed Information System for Health Care Facilities in Nigeria: A Web-based Spatial Approach
}

\author{
${ }^{1}$ Adebayo Peter Idowu \\ Dept. of Comp. Sc. \& Engr. \\ Obafemi Awolowo University, Nigeria
}

\author{
Olajide Olusegun Adeosun \\ Comp. Sc. \& Engr. Dept., \\ Ladoke Akintola University of \\ Technology, Ogbomoso, Nigeria
}

\author{
Bernard ljesunor Akhigbe \\ Dept. of Comp. Sc. \& Engr. \\ Obafemi Awolowo University, Nigeria
}

Tolulope B. Apanisile

Dept. of Comp. Sc. \& Engr.

Obafemi Awolowo University, Nigeria

\author{
Aderonke Anthonia Kayode \\ Dept. of Comp. Sc. \& Engr. \\ Obafemi Awolowo University, Nigeria
}

\section{${ }^{1}$ Corresponding Author}

\begin{abstract}
Today, the Internet has further improved the functionalities of computers. A computer and Internet based technology offering a radically different way to manage spatial data is the GIS. Its useful digital maps are needful and very useful in managing healthcare related affairs and communities as well as industry base issues. Therefore, we describe a distributive information system that uses a web-based GIS spatial approach to aid the distribution of tertiary health facilities in Nigeria. The system would assist its users to identify where health care facilities are concentrated, and how and where to locate them anytime. Some of the resources employed in the system's development include: Macromedia dreamweaver, Java Scripting, PHP, MYSQL; the WAMP server; while the UML was used for the system's design. Implementing the system showed that stakeholders were able to visualize the distribution of tertiary hospitals in Nigeria, and make useful inferential decisions with ease. Conclusively, we believe the system will aid in locating the nearest tertiary hospital, as well as help stakeholders make more informed decisions.
\end{abstract}

\section{General Terms}

Geographic information system, Software engineering, Internet, Database, Deployment architecture and WAMP, UML

\section{Keywords}

DIS, Web-base GIS, Healthcare System, Database, Spatial Modelling

\section{INTRODUCTION}

Accessibility to health care facilities has been identified as one of the major indicators of development. The importance of the availability of adequate health care facilities in providing sustainable rural development can therefore not be over-emphasized. Convergence of opinions agreed that lack of basic health care facilities have led to inefficiency in production, declining productivity, reduced life expectance and increased infant mortality rate. The health care industry in Nigeria is presently still growing unlike what is obtained in developed countries in Europe and America. This reported growth is obvious in the unavailability of good health care facilities in Nigeria. It is important to note that even when health care facility is available it is insufficient [1], [2].
Furthermore, the persistent low quality of health services that is provided in the available public health care facilities that are also inadequate has made the private sector an unavoidable choice for health care consumers in Nigeria. As a result, there is urgent need for serious intervention on the part of government in the provision of health care facilities. Government involvement is very important because to determine the rate of development in a country, one of the factors to look at is the availability of health care to people. As government intervenes their focus should be to step up the equitable distribution and accessibility of health care facilities. We expect that this would particularly enhance sustainable rural development. The argument therefore is that before government or even the private sector can intervene, there must be relevant and adequate information base that is up-todate. This information base would serve batter if it is about hospitals' locations, their concentration and specializations. This is where the use of relevant and modern Information and communication technologies (ICTs) comes in. For instance, the growth of ICTs; more particularly the Internet, has improved the ease of retrieving relevant information (about almost anything) on all aspects of life. Interestingly, this retrieval ability can occur from computer systems across the globe, such that distance is not a barrier. Likewise, it has also further broadened the already wide margins that exist between those that search for information and those that do not. The principle behind the rapid growth of information technology is that it reduces redundancy; allows more greater and accurate access to data as well as provide greater understanding of how the same data could be used in different ways.

The presence of ICTs has contributed immensely too in providing the needed platform for Information systems (ISs) (even data processing systems) to thrive. One of such ISs is the Geographical Information System (GIS). These systems GISs have become very useful tools in making strategic decisions whenever data are found to have spatial distribution [3]. The GIS technology provides support for assessment and planning in such areas as housing, healthcare, land use, natural resources, environmental surveillance and monitoring, and transportation [4]. The motivation here is that the provision of adequate health care facilities in the rural areas is tied to the availability of adequate information and data. These information and data would serve as needful and useful resource for informed and useful decision making regarding 
the situation (of health care) on ground so as to determine the areas that need attention. Necessary requirements needed in order to adopt better strategies in providing health care facilities since there are gross inadequacies would also be provided.

Therefore, this paper presents a Distributive information system (DIS) that uses a web-base GIS spatial approach to aid the distribution of tertiary health facilities in Nigeria. This system is expected to assist stakeholders in identifying where health facilities are concentrated. It is also expected to provide support with respect to rightly locating health care facilities at real time; especially to areas where serious attention is inevitable. With this system, stakeholders would be able to visualize the distribution of tertiary hospitals in Nigeria. We believe that stakeholders would be able to answer questions about the closet health care facility or hospital with the highest health care provider in Nigeria. Government would therefore benefit since the system would assist her in communicating effectively to the general public concerning the demand for more tertiary health care facilities.

The research progresses thus: Section 2.0 contains a brief literature review; section 3.0 the methodology employed in the paper; section 4.0 system design; section 5.0 system's features; and section 6.0 contains the conclusion.

\section{LITERATURE REVIEW}

Several studies have used and reported the potentials of the GIS; some of which include that of [5]; [4]; [6]; [7]; [8]; [9]; and [10]. For instance, [5] examined the location of a teaching hospital, with respect to the financial capacities of residents within locations of the teaching hospital. [4] illustrated and used the services of GIS to spatially analyze the distribution of the prevalence of asthma among school children (13-17 years) in the New York City area. In these studies the chi square inferential statistics and correlation matrix techniques were employed to analyze the spatial data. As a follow up to this, [6] developed an integrated Web-based GIS system to support policy making for conserving biodiversity. The study used spatially explicit inter-operational platform and data that are categorized into (a) base-map, (b) environmental layers, and (c) animal localities. As a result, the system is easily updated; and query cum analysis also easily carried out on situation of animal movement and habitat.

Furthermore, [7] had developed an HIV/AIDS database that is embedded in a Spatial Information Management System. Reliable data were collected to assess the status of HIV/AIDS in communities in order to demonstrate the fact that paperbased system is not reliable for inferential statistics. Earlier on [8] proposed a balanced client/server Web-based spatial analysis system that uses a Geospatial datasets for land management and its performance evaluation. Later in [9] the effect of spatial layout on nurses' movement was modelled with far greater predictability and consistency. RFID tags were used to collect time-sequence spatial data for the analysis using both the Poisson distribution function and log link function. In [10] a prototype model of Web-based GIS application for the efficient management of borehole and geological was developed to show the usefulness of spatial data analysis.

In other related studies such as that of [9]; [4]; [5]; spatial data were collected for scientific investigation and reporting. Similarly, [11] used the GIS to collect spatial data so as to examine the geographic accessibility of tertiary health services for rural population. In addition, that of [8] differs in that they argue for the need to develop web-base spatial systems. This is because the data that result from such systems do provide data patterns that are usable for informed decision making for planning purposes. In [10]; and [7] this was substantiated by the development of web-based GISs. The GIS system was developed to manage boreholes and geological data; as well as HIV/AIDS respectively. However, there are no known web-based GIS spatial system that has been applied in health related issues in Nigeria except that of [12], and [13]. Both [12], and [13] assessed the spatial distribution of health centres; and the distributional pattern of health facilities in Lokoja and Ilorin (both) in Nigeria respectively. The studies equally used the Global Positioning System (GPS) for spatial data collection. The 'Nearest Neighbour Analysis' inferential statistical tool was applied in analyzing the data collected that included the addresses of the locations of 79 health care facilities which consist of hospitals, clinics and pharmacies. Analogue maps were also collected from geospatial solutions.

However, the work reported in this paper differs since it developed a web-based GIS spatial system. The system incorporated all the capabilities of a GIS; and as such it is much more different than that of [12], and [13]. In this paper, this difference is demonstrated by offering a web based GIS for the study of the distribution of health care facilities in Nigeria; a deployment architecture; a web-based spatial system design; and an implementation unlike theirs. In a unanimous proposal, all these studies reveal that spatial data are very useful. As a result, we believe that with sound inferential statistical analysis several inferences could be arrived at for informed decision making. This paper draws from this and therefore contributes with respect to the provision of a web-base GIS for the distribution of health care facilities in Nigeria using spatial data. The results - feedback from the system would benefit for further research in that it is expected to be used in forming better strategies in the smooth distribution of health care facilities.

\section{METHODS}

In order to develop the web based spatial DIS with the sole focus of tertiary hospitals - health care facilities in Nigeria; the list and location of tertiary hospitals in Nigeria were gathered. In building the spatial database; to the map of Nigeria, layers were added. The base maps of the study area were geo-referenced, and all features were digitized layer by layer before exported to ArcView. This was followed by, first deciding on and providing the attributes of information about the web site; the dataset; and the list of hospitals in Nigeria. Secondly, the locations of hospitals in Nigeria, as well as the creation of a database were also carried out. In this database relevant tables were used to store pertinent information within the context in question. These storing processes were done automatically on the database that also served as the backend of the DIS. The datasets proposed for the web-based spatial distribution system, was added using ArcGIS software. This approach is consistent with what is obtained as it is in (e.g. [14]; [15]; [16].

The web-based aspect of the system was developed starting from the frontend using the XML and CSS using the software program editor - Macromedia Dreamweaver. Java Scripting was then used to provide the dynamism required for the website. The level of interactivity required in the user interface of the website was introduced using PHP. The PHP was also used to provide interaction between the user interface (frontend) and the database (backend) that was 
developed using MYSQL. It also enabled the passing of results back to JavaScript program and the WAMP server as a local host was used to test the suitability of the application before its upload to the internet. The Unified modelling language (UML) was used to design the system.

\subsection{The System Hardware Requirement}

In order to develop the system, both software and hardware requirements were considered. With respect to software, the ArcGIS software was deployed. This software is the ArcMap ESRI software. Its introduction provides needed mapping, tasks editing, and map-based inferential statistical analysis capabilities. In addition, using the ArcMap ESRI software, the GIS is able to map all the features that are needed on a map at real-time from satellite imagery. As a result, every necessary inferential and statistical extraction is possible. These prospects are made likely because of the invaluable components of the ArcGIS, such as the: ArcView, ArcEditor, and ArcInfo.

For instance, with the ArcView - a desktop version meant for a general (non-professional) audience; users are assisted to accomplice their desired tasks. Such tasks include the creation, integration, manipulation and visualization of cartographic databases like that of the proposed DIS [14]; [15]; [16]. The ArcEditor provides all the functionalities that add the ability to edit features. These features could be topologically integrated in a multi-user Geo-database (G-db) so that multi-user editing and visioning are possible. The third component - the ArcInfo is an ESRI's professional software. This component adds advanced geo-processing and data conversion capabilities to the general GIS functionalities. The recommended hardware requirement for the system is any complete computer system with a minimum of $80 \mathrm{~GB}$ Hard disk, and 1GB RAM. This would suffice for the server/client requirement of the proposed system. Added to this is a browser on which the system would be linked up to the Internet.

\section{SYSTEM DESIGN}

The UML provided the needed methodology to present the basic artifacts of the DIS web-based spatial system. Three basic artifacts were presented: the system's class diagram; the activity diagram; and the system's deployment architecture (see Figures 1, 2 and 3). They are meant to specify the system's design requirements. For the system deployment architecture; apart from being a design requirement, it is also meant to model the functional units of the system. The architecture (see Figure 3) reveals the packages introduced for organizing or modularizing the functional units of the system. This includes a set of major architectural patterns: The system's servers, the Client-Server and Peer-to-Peer view of the proposed system and so on. This methodology is consistent with the prescriptions of [17], [18] as reported in [19].

\subsection{The System's Class Diagram}

The class diagram (see Figure 1) comprises of 5 classes namely: QbuilderMenu (the Query Builder Menu class); UserInputMenu (the User Input Menu class), SpatialDbase (the Spatial Database class); InfrentialStat (the Inferential Statistics class); and the MapObjectMenu (the Map Object Menu class). It is important to state that as the need arises, more classes could be added; except that much caution should be exercised in order to avoid a situation of having a multiple *...* (Many to Many relationships). As much as possible for a better implementation of the system that would translate to enhanced system performance, a $1 \ldots *$ (a One to Many) relationship should be avoided. A pictorial demonstration of the system using the artifact of the class diagram is thus presented (see Figure 1).

\subsection{The System's Activity Diagram}

This UML diagram is used to model the dynamic aspect of the system in which the states represents the performance of actions. The various transitions as they are triggered along with the focuses on flows as driven by internal processing are also shown. This artifact (see Figure 2) - the activity diagram that is presented, only modelled the inferential statistics module of the system. This is a sample of as-is with respect to communicating information effectively. It also shows the procedural flow of control for various activities in the module, which is consistent with what is obtained in [20].

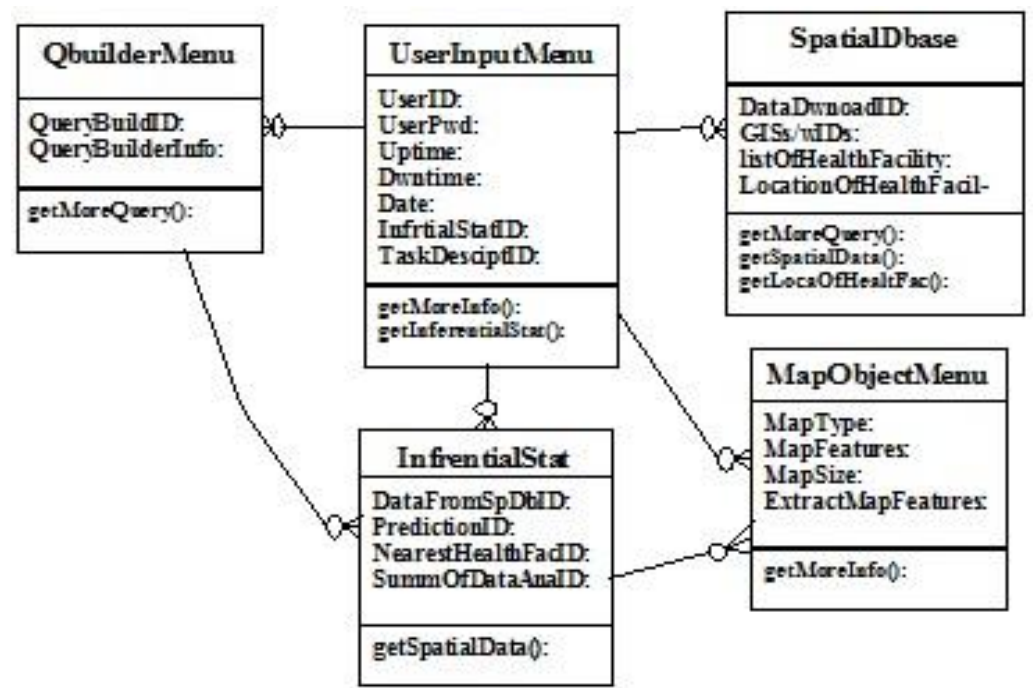

Fig 1: Class Diagram for Web-based GIS Distribution System 


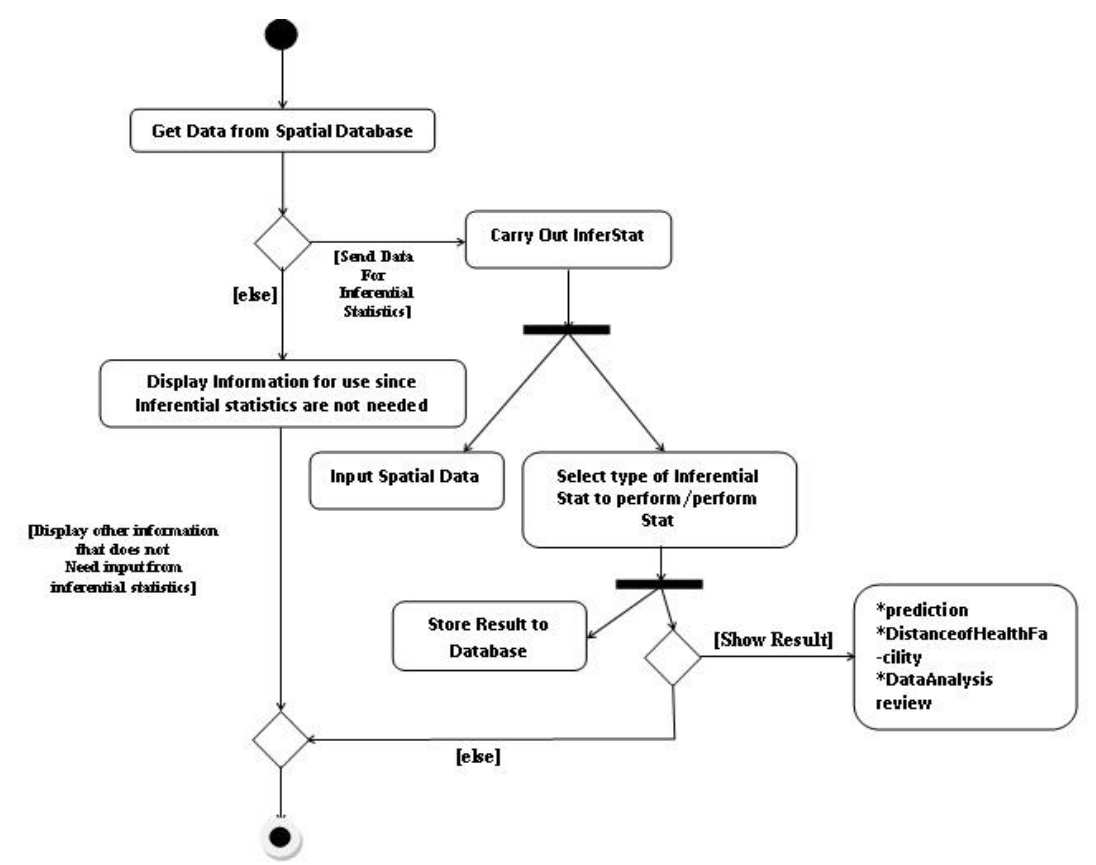

Fig 2: Activity Diagram of the Inferential Statistics Module for System

\subsection{The System's Deployment Architecture}

The system architecture is intended to describe the DIS webbase spatial system in terms of processes allocation, and with respect to the processing units and the network configuration. The components for this system were selected on the basis of the information gathered. This architectural artifact is meant to also facilitate the selection of suitable hardware and software solutions based upon the operational and system needs of GIS users.

In this paper, a centralized GIS architecture was adopted. The centralized configuration has one central GIS server that supports GIS file and database transactions. This is as opposed to a distributed GIS server which employs data replication to support a multi-department environment. In this paper (see Figure 3) a centralized GIS architecture that includes a central GIS data server that supports GIS activities over a Local area network (LAN) and Wide area network (WAN) is shown. It was adopted in order to avoid uncontrolled increase in the load on the network. As a result, client access to remote file and database servers over WAN connections would be under check, so that the performance of the network will be optimal. Additionally, while remote users that require access to the GIS data server would use a terminal client from a Microsoft Windows Terminal Server; web users would be able to access the data from the central GIS server from a web and map server easily.

\section{SYSTEM FEATURES}

\subsection{The System's Front End}

The system has two major features: The front end and the back end. The front end application is the point-and-click Graphical user interface (GUI), while the back end holds all the attribute information of the type of health care facilities available in the country. These facilities include: The General hospitals, Specialist hospitals, Teaching hospitals and the State owned hospitals on the Nigeria map. Furthermore, the next subsections provide other details regarding the system as follows.

\subsection{The System's Back End (GIS and Geo- Database)}

The map meant for the G-db was prepared using the ArcMap ArcGIS/ESRI software. To start with the development; first, the Nigeria dataset download was added as a layer to the ArcMap Document; and secondly, other layers that were created with ArcCatalogue were also added, one at a time. This was important, in order to ensure proper vectorisation that involves the process of converting raster graphics into vector graphics. In this paper, appropriate effort was made as described so far. This was to ensure that vectorisation was applied such that parts of the program where flattening would introduce overhead, without appropriate gain are avoided. This is consistency is in agreement with the prescription in [21]. This was most important; since doing so added value to the system in terms of efficient program performance than if vectorisation was just implemented.

In order to represent features on the map, layers were created and different symbols were employed to show the difference between the different layers that are there in the map. The local governments within the state on the Nigeria dataset were merged together to form a single state. As these features are extracted, provisions were made in order to have the corresponding attribute tables to be generated at the same time. Additional fields were added to these attributes field in the G-db. For example in layers Specialist hospital; apart from the three default fields another fields name, and state was added, which gave the name of the feature and state where the hospitals can be located.

In all, six layers were created, namely; General hospital, Specialist hospital, State hospital, Teaching hospital, Federal Medical Centre and Nigeria map. 


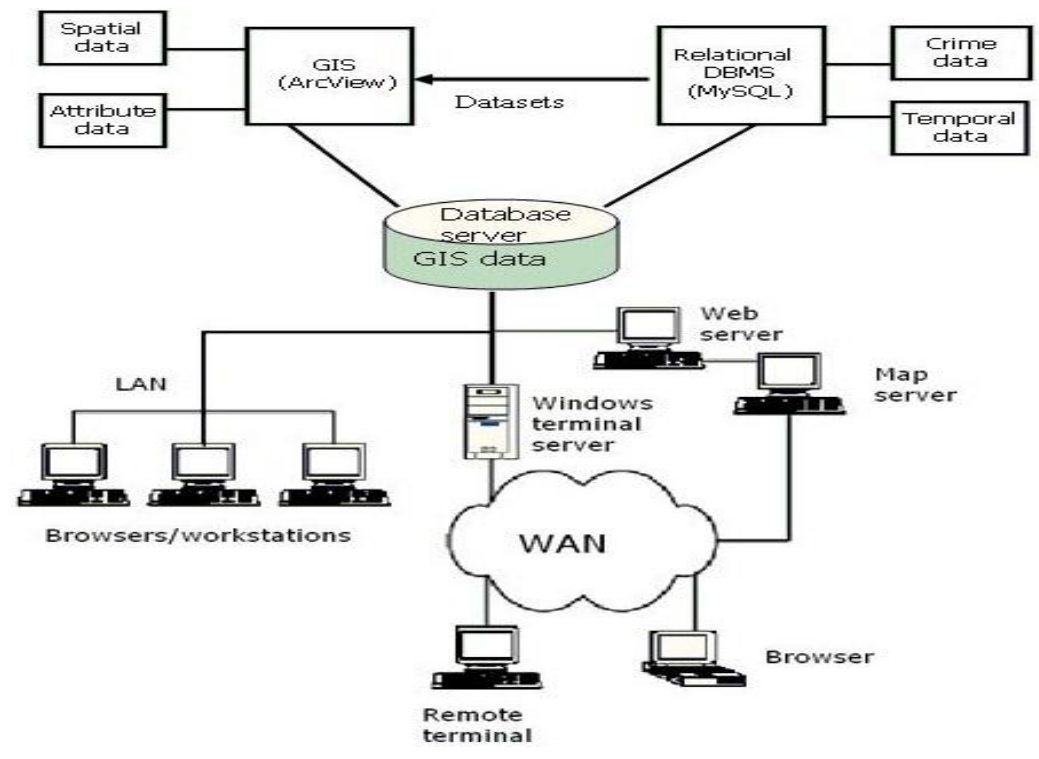

Fig 3: System Architecture for the Proposed System

\subsection{User Friendly Web Application}

To make this application user friendly, an interface that introduces the application was included. This user interface included a time that uses the time of the WAMP server that is installed on the system. This user interface was designed to be as user friendly as possible following basic interactive design principles (see Figure 4).

\subsection{Query Builder Menu}

In order to make the DIS application a web based system; the Mappetizzer software for ArcGIS was employed. This software is an adds-on to ArcGIS that had to be added to the toolbar of ArcMap on installation. The next step was to export the already completed map in ArcMap as SVG format. SVG is a scalable vector graphics and belongs to the family of graphics meant for the specifications of an XML-based file format. It was used to describe ensure that every twodimensional vector graphics - both static and dynamic (that is, interactive or animated) were accommodated. Thus, every intended image and their behaviours were easily defined in the XML text files. As a result, they - every intended image can be searched, indexed, scripted and, if required, compressed. In addition, with this SVG format, the original properties and symbologies employed in ArcGIS would be exactly what would (is intended to) be displayed to web users.

Browsers like Opera (version 9.0 or higher), Firefox (1.5 or higher), Safari (3.0 or higher) and Google Chrome support the SVG natively. For the Internet Explorer, users have to install a free browser plug-in (Adobe SVG viewer) to view SVG documents. These are the major reasons why the SVG format was adopted, among others. For better interactivity with the system, a legend was provided to assists users in knowing what each of the colours and icons used in the map represents. In order to enable a layer of the map to be click-able; a click on the check box in front of the layer's name is all the user needed. Each layer shows where the various hospitals are located on the map as the name of the legend implies. By default all the check boxes are checked, but they could be unchecked too if desired.
Basically, users can carry out any level of queries on any of the attributes in the G-db. The query tool can be found on the legend part of the web page. For easy use of the query tool a checkbox is associated with each legend and two other icons the Attributes icon (Ai) and the Query Tool icon (QTi). When the QTi is clicked, a new window named query builder would open up. The query builder window shows each of the layers and the query that could be carried out on them. When the query is built and executed, the portion affected on the map would be highlighted in red (since red is the colour specified at the design stage). An example of the query builder window is as presented in Figure 5.

The other icon - the Ai which is associated with each layer of the legend is the attributes icon. This icon makes it possible for attributes of the current layer as it is in the G-db to be displayed on the web interface and be sent to a printer if a hard copy is desired.

\subsection{Map Object/Area Menu}

Another tool present is the overview map object. This object helps to show the whole map area. This is necessary; otherwise it might not be know at a glance. This is because the map's original extent is bigger than the display window and cannot be seen at once. So, the overview window helps to show the users the full extent of the map. On the overview object, there is a 'mask' hovering over it that shows the portion of the map to be currently displayed. This mask could be adjusted as desired in order to change the part of the map users wish to display.

The overview map could have been displayed on another window, but the result arrived at as a result of the system implementation show that it is better if displayed on the current window. An example of the overview map in details is thus presented. The map is a typical distributive output, showing the location of hospitals in Nigeria (see Figure 6). The activities on the menu beside the map are aspects of the project that are left for future work; except the activity of save as. As earlier stated this provided another easy way to send a job for printer if a hard copy is what is needed. 


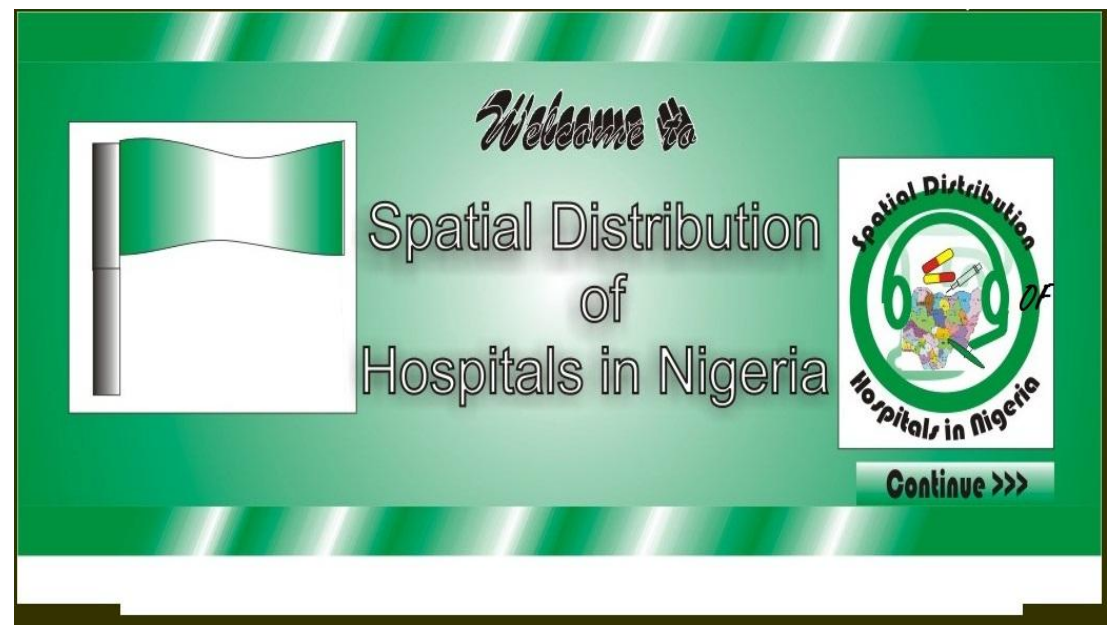

Fig 4: User friendly interface

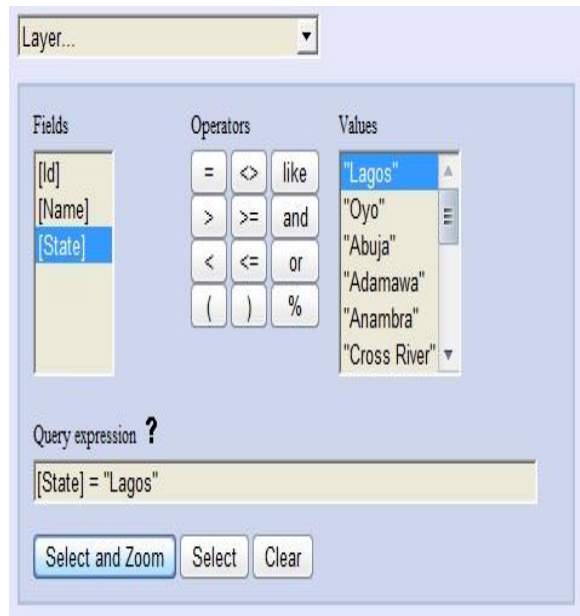

$\uparrow$ Badc to guery builder

\begin{tabular}{|c|c|c|c|}
\hline \multicolumn{4}{|c|}{$\begin{array}{l}\text { Note: } \\
\text { Move cursor over the rows to highighit the objects on the map. }\end{array}$} \\
\hline & Id & Name & State \\
\hline 0 & .99999 & Talent Specialist Hospita, Gorron estate, Ipaja & Lagos \\
\hline 0 & 0 & Sinai Specialist Hospital, 19a Military Street, Onilikan & Lagos \\
\hline 0 & 0 & Shepherd Specialist Hospital, 4th Avenuve, E Close, Festac Town & Lagos \\
\hline 0 & 0 & St Claire Specialist Clinic 44 Apena St, Survelere & Lagos \\
\hline 0 & 0 & Rapha Specialist Hospital, Royal Cross Hospital, 24 Crown Estate, Lelkki Perinsula & Lagos \\
\hline 0 & 0 & National Orthopaedic Hospita, Igboobi & Lagos \\
\hline 0 & 0 & M. Zion Specialist Clinic, Adeolus Street, Survilere & Lagos \\
\hline 0 & 0 & Created with Mappetizer for ArcGIS by uismedia & Lagos \\
\hline 0 & 0 & Lifegate Specialist Hosppital, off Acme Road, Oggla & Lagos \\
\hline 0 & 0 & Leo Specialist Hospital, Balogn Street, Lagos & Lagos \\
\hline 0 & 0 & Harana Specialist Hospital Ltd, Akerele Street (Teslim Fatusi Street), Survlere & Lagos \\
\hline
\end{tabular}

Fig 5: A Query builder window

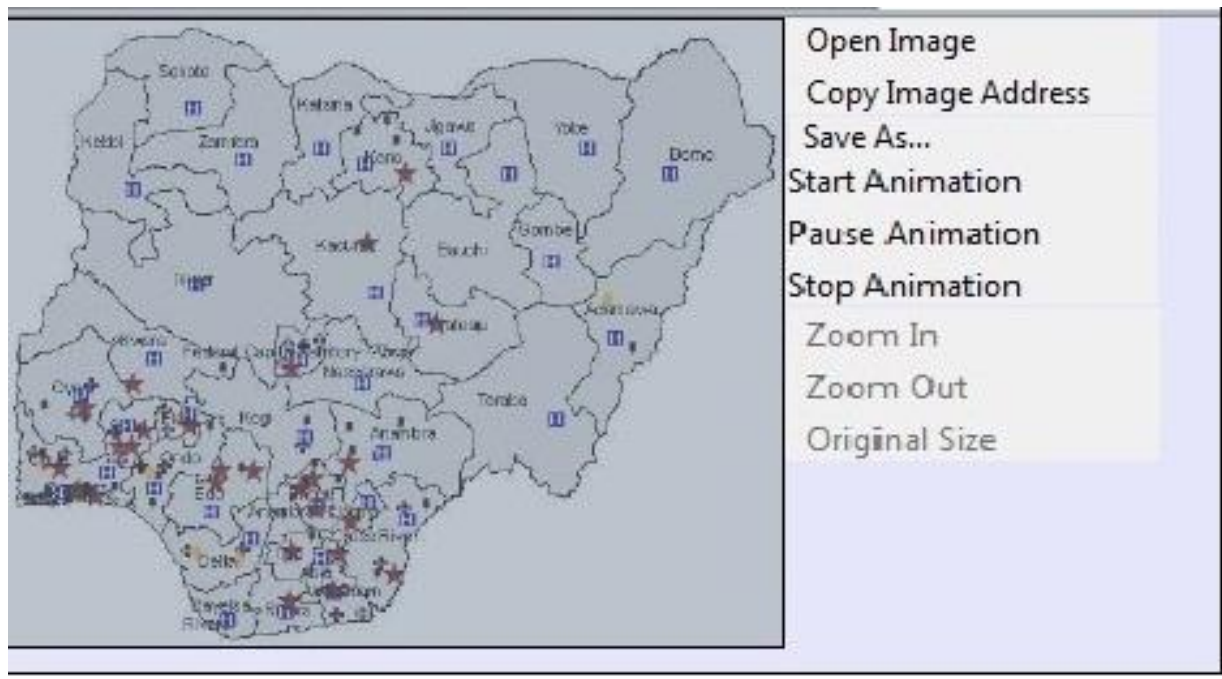

Fig 6: A Distributive Output as result of DIS use Showing hospital distribution 
This map area is the actual map displayed (see Figure 6). The display is a function of the presence of the legend as enabled on the legend area; the scale used; and the tools resident in the toolbar. Figure 6 show a clear example of the map displayed in the map area. The map (see Figure 6) can be displayed independently be being printed if needed.

\section{CONCLUSION}

In this study, an example DIS that is Web-based is presented. It uses spatial data to aid the management of the distribution of health care (hospital) facilities in Nigeria. The DIS essentially adopts the Internet/web-base technology because the efficiency of data usage is very important considering the peculiarity of the case study country (Nigeria) that is a fast developing and growing economy. Furthermore, the need to have uninterrupted access to data and other related result, also necessitated the use of internet technology. It is believed that from any part of the country, there should be an unhindered access to data since the saving of lives is what is at stake. This prototype DIS was successfully designed using the UML, and implemented using the ArcGIS software. The web-based aspect was handled using the XML and CSS software program editor of the Macromedia Dreamweaver. Other software resources such as Java Scripting, PHP, and MYSQL contributed immensely in introducing the dynamism/interactivity (business logic) required for the webbased system and between the user interfaces, and the database (backend).

As a result of this the purpose of this paper with respect to safely, timely and easily generating information was met. We therefore believe that this system would allow Nigerians to be able to view the location of their desired hospital - health care facilities on a map as easily as possible. Even, government agencies and their officers would be able to easily view and ascertain areas that need more hospitals to be established. The result of the system implementation showed that all relevant and intended maps and corresponding information were easily created and generated. Thus, making it obvious that there are more hospitals at the western part of Nigeria as compared to the eastern part; and that the lesser hospitals (in terms of the type of health care services) are situated in the northern part of Nigeria.

The spatial distribution of hospitals in Nigeria using a DIS Map-suite, like other software, is not without its limitations. First, there is dearth of information about the federal hospitals in Nigeria due to lack of detailed documentation on the part of stakeholders, who still depend majorly on manual means of record keeping. The second limitation is that this study only modelled the State government owned hospitals. Finally, the remaining activities in the Menu shown in Figure 6 would be considered for future work. Effort can also be made to consider viable platforms on which the DIS system can be easily connected to existing GIS applications in order to further strengthen the capability of the application. Conclusively, an unfettered access to available information about the distribution of hospitals in a country can efficiently impact on the delivery of health care services. This is particularly necessary for a developing economy like that of Nigeria which is fast and very competitive in Sub-Sahara Africa, where timely diagnosis of diseases and its treatment is important for existing health care providers to survive.

\section{ACKNOWLEDGMENTS}

Our sincere thanks go to all who contributed to this research effort one way or the other. And to Obafemi Awolowo University and Ladoke Akintola University of Technology, Nigeria we say thank you for the space given to us to do this research.

\section{REFERENCES}

[1] Perry, B., and Gesler, W. (2000). Physical Access to Primary Health Care in Andean Boliva. Soc.Sci Med 2000, 50:1177-88.

[2] Olutola .M. O and Oloruntoba .S. O, (2009). An information Databank Framework for

the Health care Industry in Nigeria, International Journal of Computing and ICT Research, Vol. 3, No. 2, December 2009.

[3] Bunch, M.J., Kumaran, T.V., and Joseph, R. (2012). Using Geographic Information Systems (GIS) For Spatial Planning and Environmental Management in India: Critical Considerations. International Journal of Applied Science and Technology Vol, 2(2), pgs 40-54

[4] Rob, M.A. (2003). Applications of Geographical Information Systems in Understanding the Spatial Distribution of Asthma. Informing Science Journal, Volume 6, pgs 89-99.

[5] Abegunde, A. A., and Adedigba, A.M. (2011). Teaching Hospitals in African Nations: Infras-tructure for Regional or Local Community Development? The African Symposium, Vol. 11, No. 2, pgs 49-59.

[6] Doko, T. (2009), Web-based GIS development as a policy making support system for conserving biodiversity: Case study in Fuji-Tanzawa region, Japan, The 33rd International Symposium on Remote Sensing of Environment, Volume I \& II, pp. 344-347, Stresa, Italy, 48 May 2009. (ISBN: 978-0-932913-13-5)

[7] Busgeeth, K., and Rivett, U. (2004). The use of a spatial information system in the manage-ment of HIV/AIDS in South Africa. International Journal of Health Geographics, 3:13 doi:10.1186/1476-072X-3-13

[8] Vatsavai, R.R., Burk, T.E., Wilson, B.T., and Shekhar, S. (2000). A Web-based browsing and spatial analysis system for regional natural resource analysis and mapping. In Proceedings of 8th ACM Symposium on GIS, Washington, D.C., USA, pgs 95-101.

[9] Heo, Y., Choudhary, R., Bafna, S., Hendrich, A., and Chow, M.P. (2009). A Modeling Approach for Estimating the Impact of Spatial Configuration on Nurses' Movement. In Proceedings of the 7th International Space Syntax Symposium, Eds Daniel Koch, Lars Marcus and Jesper Steen, Stockholm: KTH, Ref. 041

[10] Chang, Y.-S., and Park, H.-D. (2004). Development of a web-based Geographic Information System for the management of borehole and geological data. Computers \& Geosciences 30, pgs 887-897

[11] Beedasy, J. (2010). Rural Designations and Geographic Access to Tertiary Healthcare in Idaho. The Online Journal of Rural Research and Policy. Vol. 5 (2): 1-21.

[12] Alabi, M.O. (2011). Towards Sustainable Distribution of Health Centers Using GIS: A Case Study from Nigeria. 
American Journal of TROPICAL MEDICINE \& Public Health, 1(3): 130-136.

[13] Jimoh, H.I. and Azubike, J.O. (2012). The Roles of Geographic Information System on the Distributional Pattern of Health Care Facilities in Ilorin, Kwara State of Nigeria. International J. Soc. Sci. \& Education, Vol. 2 (2), 224-235.

[14] Board, C. (1990) Report of the Working Group on Cartographic Definitions. Cartographic Journal, 29(1), 65-69.

[15] Tomlin C.D. (1990) Geographic Information Systems and Cartographic Modelling, Pretentice Hall.

[16] Fadahunsi, J.T. (2011). Application of Geographical Information System (GIS) Technology to Tourism Management in Ile-Ife, Osun State, Nigeria. The Pacific Journal of Science and Technology, Vol. 12 (2), pgs 274283.
[17] Pender, T. (2003). UML Bible, John Wiley and Sons, 2003.

[18] Bennet, S., McRobb, S., and Farmer, R (2003). ObjectOriented Analysis and Design using UML, McGraw-Hill, 2003.

[19] Ojo, A., and Estevez, E. (2005). Object-Oriented Analysis and Design with UML: A Training Course. e-Macao Report 19, V 1.0, UNU-IIST.

[20] Bell, D. (2003). UML basics; Part II: The activity diagram. In the Rationale Edge. Retrieved from http://www.therationaledge.com/content/sep_03/f_umlbas ics_db.jsp on 13/10/ 2012 @ 5:46 pm

[21] Keller, G., Chakravarty, M.M.T., Leshchinskiy, R., Lippmeier, B., and Jones, S.P. (2012). Vectorisation Avoidance. In Haskell'12, Copenhagen, Denmark and ACM 2012 978-1-4503-1574-6/12/09. Retrieved from http://research.microsoft.com/en- us/um/people /simonp/papers/ndp/vectorisation-avoidance-hw12.pdf 